\title{
La eVolución del empleo en Chile 1990-2010: UN RECÁLCULO
}

\section{Oscar Landerretche Gacitúa}

Instituto de Asuntos Públicos, Universidad de Chile

oscar.landerretche@iap.uchile.cl

En el artículo se demuestra que, eliminando distorsiones estadísticas, Chile habría logrado crear unos 320.000 empleos en 2010, es decir, 160.000 menos que lo que ha informado el Instituto Nacional de Estadísticas, entidad que no ha sido suficientemente clara en cuanto al carácter "referencial" que le confiere a las cifras del 2009, sobre la base de las cuales se calcula el crecimiento en 2010. La ausencia de cifras oficiales y crébles es algo inédito y afecta los cálculos sobre la evolución de la productividad del trabajo (reduciendo su crecimiento), lo cual incide sobre negociaciones salariales de amplio espectro, tales como las del salario mínimo o los reajustes de sueldos del sector público. También se argumenta que la expansión del empleo parece presentar problemas de "calidad" de los empleos generados, tanto por el predominio del autoempleo como por el bajo desempeño de la productividad.

Palabras Clave: empleo, estadísticas, políticas públicas, Chile.

\section{The eVolution of employment in Chile 1990-2010: A RECALCULATION}

The article shows that, by eliminating statistical distortions, Chile created 320.000 new jobs in 2010, i.e, 160.000 less than was reported by the National Statistics Institute, an organization which has been insufficiently clear regarding the referential nature of its 2009 figures used to calculate growth in 2010. The lack of official and credible figures is unprecedented and affects calculations of the evolution labor productivity (reducing growth), which in turn affects wideranging wage bargaining, such as those concerning the minimum wage and the adjustments of public sector salaries. It is also argued that the increase in employment suffers from problems of "quality", arising from the preponderance of self-employment and low productivity.

Keywords: employment, statistics, public policies, Chile. 


\section{El contexto: los compromisos del actual Gobierno}

En la introducción de su Programa de Gobierno, el actual Presidente de la República, Sebastián Piñera, destaca la importancia del compromiso sobre creación de empleos en el marco del Programa completo:

El Programa será la carta de navegación del cambio que proponemos para Chile. Se trata de un cambio en paz, con unidad, armonía y tolerancia. Este cambio sólo busca lo mejor para los chilenos: lograr ser un país desarrollado, superar la pobreza y apoyar la clase media abandonada. Él muestra cómo se construirá una sociedad de oportunidades para todos. Un millón de nuevos empleos, una cultura a favor del emprendimiento y la innovación serán pilares fundamentales para ello. Nuestro Programa construye también el camino para alcanzar una real sociedad de seguridades. Una sociedad donde los chilenos no se sientan abandonados sino que acogidos, acompañados y potenciados. En suma, una sociedad más justa que elimine la indigencia y le asegure a cada familia un ingreso digno.

Queremos construir también un país con valores en el cual respetemos y protejamos la vida, y dignifiquemos y reforcemos a la familia. Donde cuidemos el medio ambiente, promovamos la cultura, y el amor a la Patria. Así perfeccionaremos y profundizaremos la libertad, la Democracia y el Estado de Derecho. Este es el Chile que queremos y soñamos (Piñera, 2009:9).

El objetivo analítico de este trabajo es evaluar el avance de este compromiso gubernamental fundamental, la creación de un millón de nuevos y buenos empleos ${ }^{1}$, desde el punto de vista de los resultados prometidos, no de la gran variedad de medidas anunciadas y sus respectivos avances.

Consideramos preferible hacerlo así, porque muchas de las medidas pueden ser discutibles en su efectividad o eficiencia y porque es muy dificil, en un período corto, diferenciar sus efectos particulares. Además, muchos de sus resultados tomarán tiempo en manifestarse completamente.

Este compromiso central está reiterado en documentos posteriores al terremoto del 27 de febrero de 2010, evento de gran envergadura que, sin duda, podría haber justificado cambios en algunas de las prioridades ${ }^{2}$.

\footnotetext{
Señala el Presidente:"Para superar los problemas indicados planteamos como meta para los próximos cinco años la creación de un millón de nuevos y buenos empleos, con salarios justos, posibilitándole un mejor nivel de vida a miles de chilenos que sólo buscan que se les brinde la oportunidad de trabajar" (Piñera, 2009: 64-65).

2 Hemos analizado este tema en Landerretche Gacitúa (2010: 39-67).
} 
¿Cómo crearemos esos doscientos mil empleos al año? El crecimiento del 6\% anual generará unos 150.000 puestos de trabajo al año. Necesitamos, por tanto, buscar otras fórmulas que nos permitan crear 50.000 empleos adicionales (...) En primer lugar, vamos a capacitar a 5 millones de trabajadores durante nuestro gobierno, para que puedan acceder a mejores empleos y mayores remuneraciones (...) Segundo, daremos un nuevo trato a las PYMES, que son las que generan el $80 \%$ del empleo en Chile. No sólo eximiéndolas del impuesto de primera categoría sobre las utilidades reinvertidas, sino también, evaluando el impacto que toda nueva política, ley o reglamento tenga sobre ellas. También tengo hoy la satisfacción de anunciar un proyecto de ley que reducirá de 27 a sólo 16 días los trámites necesarios para crear una nueva empresa, con su consiguiente ahorro de costos (...) En tercer lugar, vamos a promover el teletrabajo y trabajo desde el hogar (...) Cuarto, diseñaremos durante este año un Bono de Intermediación Laboral, financiado por el Estado, para ayudar a encontrar trabajo a personas de baja calificación (...) En paralelo, ya adjudicamos la puesta en marcha de una Bolsa Nacional de Empleo, que permitirá a más de 7 millones de trabajadores conocer mejores opciones laborales (...) En quinto lugar, perfeccionaremos el seguro de desempleo (...) Sexto, impulsaremos una verdadera cultura del emprendimiento, la innovación y la creatividad, que nos permita generar 100.000 nuevos emprendimientos en los próximos 4 años (...) Por último, y lo más importante, nuestro gobierno va a respetar y hará respetar los derechos de los trabajadores (Piñera, 2010:14-16).

En materia de Empleo, proponemos incrementar en 390 mil millones de pesos el presupuesto del Ministerio del Trabajo. Esto permitirá dar un paso importante hacia la meta de capacitar a 5 millones de trabajadores durante nuestro Gobierno. También permitirá triplicar la cobertura de las Oficinas Municipales de Intermediación Laboral, llegando a 300 comunas. Además considera los recursos para entregar subsidios a la contratación de 140.000 jóvenes, un 40\% más que en 2010" (Mensaje 331-358 de 2010, pág. 2).

Pero en los últimos años la productividad de la economía nacional cayó sostenidamente. Perdimos la capacidad de crecer y de crear empleo vigorosamente. El primer compromiso de nuestra agenda es revertir esta situación, y estamos destinando todos nuestros esfuerzos a conseguirlo (...) Llevamos cinco meses de crecimiento sostenido en torno al 7\%. Esto significa que hay más y mejores empleos, más y mayores oportunidades para todos los chilenos, en especial para los más necesitados y para la gran clase media de nuestro país. Esto se ha traducido ya en la creación de más de 200.000 empleos en menos de 6 meses, de los cuales cerca de 130 mil han sido para mujeres (Ministerio de Hacienda, 2011: 1-2).

Estrictamente hablando, los compromisos sobre creación de empleos de buena calidad aparecen directamente vinculados a los compromisos sobre crecimiento e incremento de la productividad: 
Nos proponemos que para el año 2018, bicentenario de la batalla de Maipú, con la cual se inicia la real y definitiva independencia nacional, Chile tenga un ingreso por habitante superior a los 22.000 dólares al año y de esta forma iguale las condiciones de vida de los países del sur de Europa (Piñera, 2009:15).

En realidad, el compromiso no parece bien planteado porque según los registros del Fondo Monetario Internacional (FMI, 2011) los países del "sur de Europa" como España, Italia, Grecia, Eslovenia, Eslovaquia, Portugal e incluso Chipre, Malta e Israel ya están por encima de ese "nivel" en 2010, la mayor parte de ellos significativamente por encima (más de un 30\%) y sólo Croacia parece "alcanzable", incluso con escenarios de estancamiento prolongado en países como Portugal y Grecia.

Por lo demás, el FMI no pronostica, para Chile, el ritmo de crecimiento que el Gobierno se ha puesto como meta y ello indica que se requiere un gran esfuerzo deliberado para lograrlo.

Por cierto, en documentos posteriores, la meta del Gobierno se ha "relajado" y ya no se plantea alcanzar en el 2018 las condiciones de vida del "sur de Europa", sino alcanzar las condiciones de vida que tenían en 2009 países como Portugal o la República Checa:

Nuestra meta es crecer al 6\% promedio anual, y así alcanzar el desarrollo en 8 años, superando el ingreso per cápita que actualmente tienen países como Portugal o la República Checa (Piñera, 2010:13).

Como Gobierno, estamos convencidos que Chile puede conseguir el sueño de llegar a ser un país desarrollo en 2018, pero para ello debemos lograr crecer en promedio a $6 \%$ anual hasta entonces. Esto significa alcanzar en el año 2018 niveles de PIB per cápita similar al exhibido por Portugal o la República Checa en 2009 (Ministerio de Hacienda, 2011:13).

¿Puede ser atribuido este cambio de metas a los efectos del terremoto del 27 de febrero de 2010? No parece razonable para una meta de largo plazo.

Aun descontando - para siempre- un 3\% de la meta por efecto pérdida de stock de capital debido a terremoto ${ }^{3}$, lo cual es discutible desde el punto de vista de la 
experiencia internacional, porque este tipo de desastres, en países del nivel de desarrollo de Chile, tienden a generar más inversión y más crecimiento (Albala-Bertrand, 1993) ${ }^{4}$, la meta no tendría por qué haber cambiado significativamente, dado que se mantiene el objetivo de crecer a un ritmo de un $6 \%$ anual, lo cual implica que el PIB per capita crecería a un 5,1\% anual promedio en el período 2010-2018, de acuerdo a las proyeccciones de población del INE.

La relación directa entre las metas de crecimiento, productividad y empleo queda más clara en el siguiente Cuadro, preparado por el Ministerio de Hacienda, en que se resumen las metas de la conducción económica.

\section{Cuadro 1 \\ Objetivos Estratégicos para el período 2010-2013}

\begin{tabular}{|c|l|}
\hline \multirow{2}{*}{ Crecimiento } & $\begin{array}{l}\text { Alcanzar un crecimiento promedio del PIB de 6,0\% en el } \\
\text { período 2010-2013 }\end{array}$ \\
\cline { 2 - 3 } & $\begin{array}{l}\text { Aumentar la inversión, desde el 21,4\% actual, hasta un 28\% del } \\
\text { PIB al año 2014 }\end{array}$ \\
\cline { 2 - 3 } Empleo & $\begin{array}{l}\text { Aumentar la inversión en I+D, desde el 0,4\% actual, hasta un } \\
0,6 \% \text { del PIB al } 2013 \text { y 0,8\% al 2018 }\end{array}$ \\
\hline & $\begin{array}{l}\text { Crear } 100.000 \text { nuevos emprendimientos en el periodo 2010 } \\
2013\end{array}$ \\
\cline { 2 - 2 } & $\begin{array}{l}\text { Crear 200 mil nuevos empleos promedio al año, durante el } \\
\text { período 2010-2014 }\end{array}$ \\
\cline { 2 - 2 } & Capacitar a 5 millones de trabajadores en el periodo 2010-2013 \\
\hline
\end{tabular}

Fuente: Ministerio de Hacienda, 2010

Una novedad de este Cuadro reside en que mientras en el Programa de Gobierno se proponía incrementar el gasto en I+D desde un $0,7 \%$ del PIB en 2009 a un 1,2\% del PIB en 2013, aquí se "revisan" las cifras que son el punto de partida y se plantea una meta mucho menos ambiciosa. ${ }^{5}$ Este aspecto no es menor a largo plazo si ello

de dólares de los EEUU, es decir, entre un 1,6\% y un 4,4\% del stock de capital productivo estimado a través de la metodología de inventarios perpetuos" (Landerretche Gacitúa, 2010:44).

4 Por su parte, Loayza et al. (2009:22) encuentran que las tormentas y los terremotos tienden a tener un efecto neto positivo sobre el crecimiento, a mediano plazo (en un contexto analítico de pleno empleo) debido a que la reducción del stock de capital por persona empleada aumenta, posteriormente, el ritmo de inversión. Este trabajo desagrega por tipos de países, más o menos desarrollados, tipos de desastres y sectores productivos afectados.

5 Comparar, por ejemplo, con: "Por ello, vamos a aumentar el nivel de inversión en I+D desde los niveles actuales de $0,7 \%$ del PIB a 1,2\% del PIB a fines del Gobierno y sentar las bases para que 
implica reducir el esfuerzo para incrementar la productividad que, como veremos, lejos de repuntar, como era el objetivo planteado, se ha manifestado de manera negativa.

Estando claros los compromisos y las metas, volvemos al objetivo analítico de este trabajo que es evaluar el avance del compromiso, fundamental del Gobierno del Presidente Piñera en lo que se refiere a creación de empleos de buena calidad.Veamos primero las cifras sobre cantidad de empleo.

\section{El análisis de las cifras}

En diversos reportes mensuales del Taller de Política Públicas del INAP, se señaló, durante el $2010^{6}$, que las series de empleo, de la Nueva Encuesta Nacional de Empleo (NENE), que sustituyó a la anterior encuesta en marzo de 2010, se comportan de manera bastante extraña si se contrastan su primeras seis cifras mensuales (los primeros seis trimestres móviles) con las siguientes seis cifras (que completan un año de registros). Ello es evidente cuando se compara la nueva serie con la de la anterior Encuesta Nacional de Empleo (ENE).

Como puede verse en los gráfico 1 y 2 (permiten una perspectiva más amplia), la nueva encuesta "comparte" niveles con la anterior al comienzo, para distanciarse, más tarde, de manera radical. Como resultado de ello las dos encuestas "cuentan" una historia muy distinta sobre lo ocurrido durante el año 2009.

pueda duplicarse nuevamente en los siguientes 8 años, alcanzando el 2,4\% del PIB a finales del 2021" (Piñera, 2009:29). “Aumentaremos de 0,7 a 1,5 \% del PIB la inversión en ciencia y tecnología, con alianzas y esfuerzos compartidos entre el Estado, las universidades e institutos y la empresa privada" (Piñera, 2010: 14).

${ }_{6}$ Los informes puede encontrarse en http://www.inap.uchile.cl/taller-politicas-publicas.html 


\section{Gráfico 1}

Comparación entre encuestas de empleo del INE (2009-2010) Anterior (ENE) y nueva (NENE). Miles de personas ocupadas.

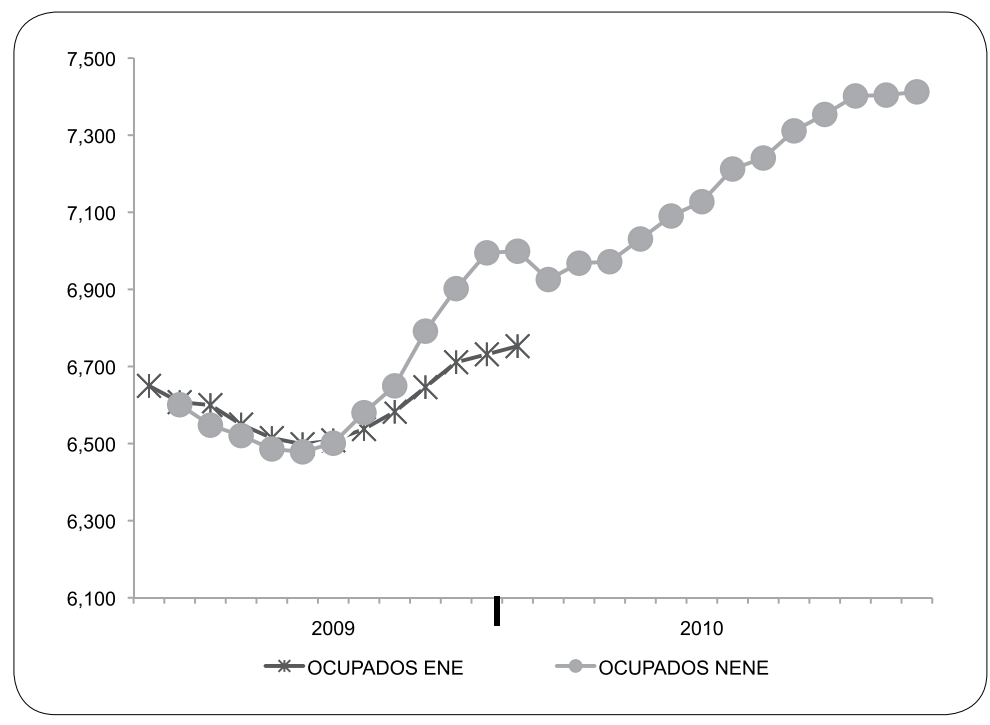

Fuente: Informaciones del INE

Gráfico 2

Comparación entre encuestas de empleo del INE (2006-2010)

Anterior (ENE) y nueva (NENE). Miles de personas ocupadas

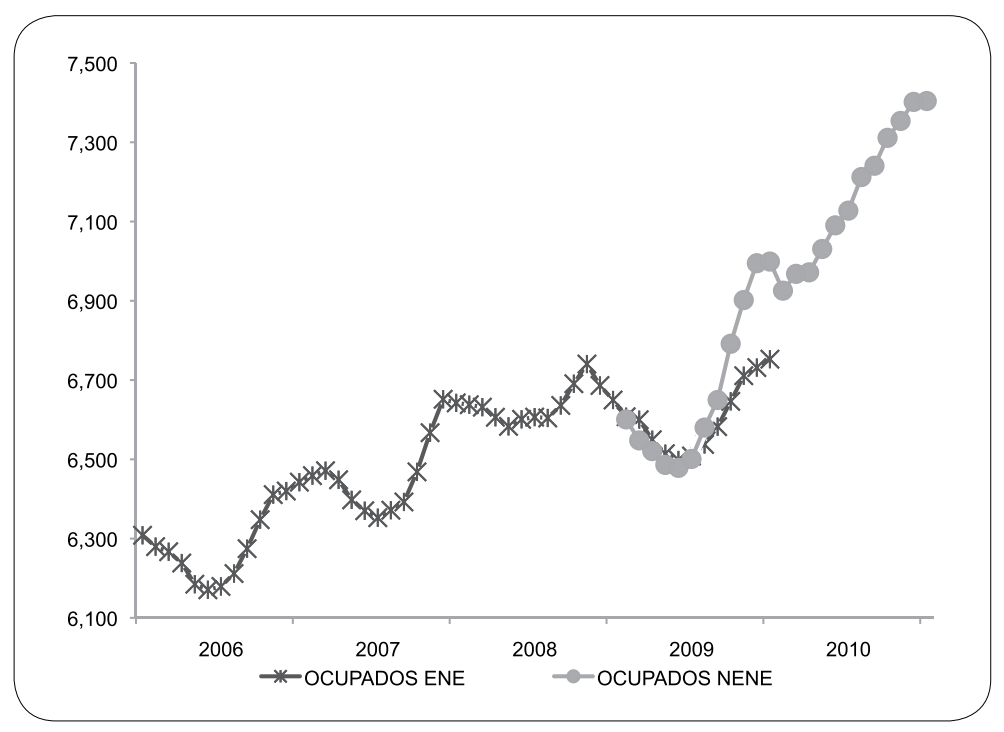

Fuente: Informaciones del INE 
Dicho de otra manera, la nueva encuesta (NENE) "reescribe" la evolución del empleo durante 2009. La nueva encuesta muestra un enorme dinamismo del empleo en el segundo semestre, con un incremento del orden de los 500.000 empleos entre mediados del año y fines del año, una cifra inédita e inconsistente con el modesto crecimiento de la economía.

Esto hace que las variaciones en 12 meses de la nueva serie sean mucho mayores que las de la serie anterior, especialmente durante el primer semestre de 2010, generándose un "salto" en el ritmo de creación de nuevos empleos que parece tener un componente "espurio" porque se produce en el primer reporte de la nueva serie, que corresponde al trimestre móvil enero-marzo 2010, que contiene el impacto inicial del terremoto del 27 de febrero, que debería ser negativo, a pesar de lo cual se observa un tremendo salto positivo, lo que esta expresado en el siguiente gráfico.

\section{Gráfico 3}

Comparación entre ENE y NENE

Porcentaje de crecimiento del empleo en 12 meses

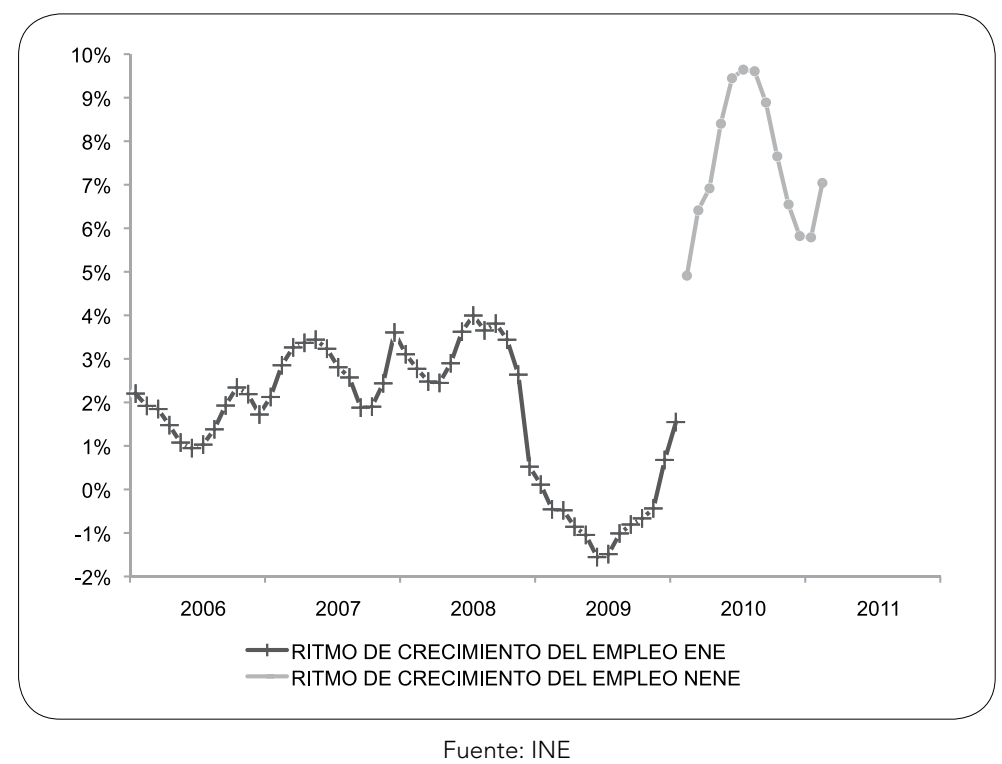

En realidad, se podría aceptar que una nueva metodología midiera un mayor número de ocupados, es decir, que los "niveles" de esta u otra variable incluyan más personas debido a cambios en las definiciones. Sin embargo, resulta mucho menos razonable suponer que la nueva encuesta vaya a dar como resultado cambios tan grandes en 
el ritmo porcentual de generación de empleo y precisamente en el trimestre móvil en que la serie debiera capturar el efecto del terremoto en el empleo ocurrido en el mes de marzo de 2010.

Según ambas series de cifras (ENE y NENE), el empleo nacional total era prácticamente el mismo en el primer y segundo trimestres de 2009 (trimestres eneromarzo y abril-junio de 2009), siendo levemente menor el registro de la NENE (ver cuadro 2). Sin embargo, en los dos trimestres posteriores (julio-septiembre y octubre-diciembre) el nivel de empleo es significativa y crecientemente distinto, alcanzando una sorprendente diferencia de casi doscientas mil personas entre ambas series, en el último trimestre de 2009.

Cuadro 2

Empleo total en miles de personas

\begin{tabular}{|l|c|c|}
\cline { 2 - 3 } \multicolumn{1}{c|}{} & ENE & NENE \\
\hline enero-marzo 2009 & 6.608 & 6.601 \\
\hline abril-junio 2009 & 6.514 & 6.486 \\
\hline julio-septiembre 2009 & 6.538 & 6.580 \\
\hline octubre-diciembre 2009 & 6.711 & 6.902 \\
\hline
\end{tabular}

Fuente: INE

El INE ha resuelto esta anomalía de manera bastante insatisfactoria, incluyendo un aviso en uno de los sectores de su página $w^{2} b^{7}$, el cual reconoce, en buena medida, la "anomalía" señalada:

Las cifras de año 2009 son sólo referenciales. No son cifras oficiales, sino resultado del levantamiento paralelo en que se instalaron paulatinamente los procesos operativos, técnicos, y del cuestionario de la Nueva Encuesta Nacional de Empleo. La variabilidad de estas cifras limita su uso sólo para fines analíticos.

Es una lástima que el indicador requerido para medir adecuadamente una de las metas o compromisos centrales del Gobierno, presente esta "anomalía" que dificulta las comparaciones con la historia anterior. En realidad, lo que el INE ha hecho, hasta ahora, es desentenderse de la obligación que tenía de proveer algún empalme

Ver Nueva Encuesta Nacional de Empleo (NENE), Cifras Referenciales 2009 en la dirección: http://www.ine.cl/canales/chile_estadistico/mercado_del_trabajo/nene/series_referenciales.php 
adecuado que permitiera contar con series históricas de empleo (nacional y por regiones, total y por rama de actividad económica, y por categoría de ocupación).

Al reconocer que no se hizo una recolección paralela con las dos metodologías, durante 2009, cualquier análisis queda ahora sujeto a la aceptación de los supuestos en que se base, porque a la serie le falta un dato crucial, el crecimiento del empleo entre 2009 y 2010 , que el propio INE señala que no se puede calcular técnicamente porque sus datos 2009 no son oficiales sino sólo "referenciales". ${ }^{8}$

No es creíble que el ritmo de creación de empleos haya superado los 600.000 anuales, durante el invierno de 2010, con tasas de crecimiento del empleo superiores a $9,5 \%$ anual, tasas que resultan ser completamente inéditas en la historia económica reciente de Chile (ver gráfico 4) y que implican, además, una caída notable y poco creíble, en la productividad, como lo veremos más adelante.

\section{Gráfico 4}

Crecimiento anual del empleo nacional

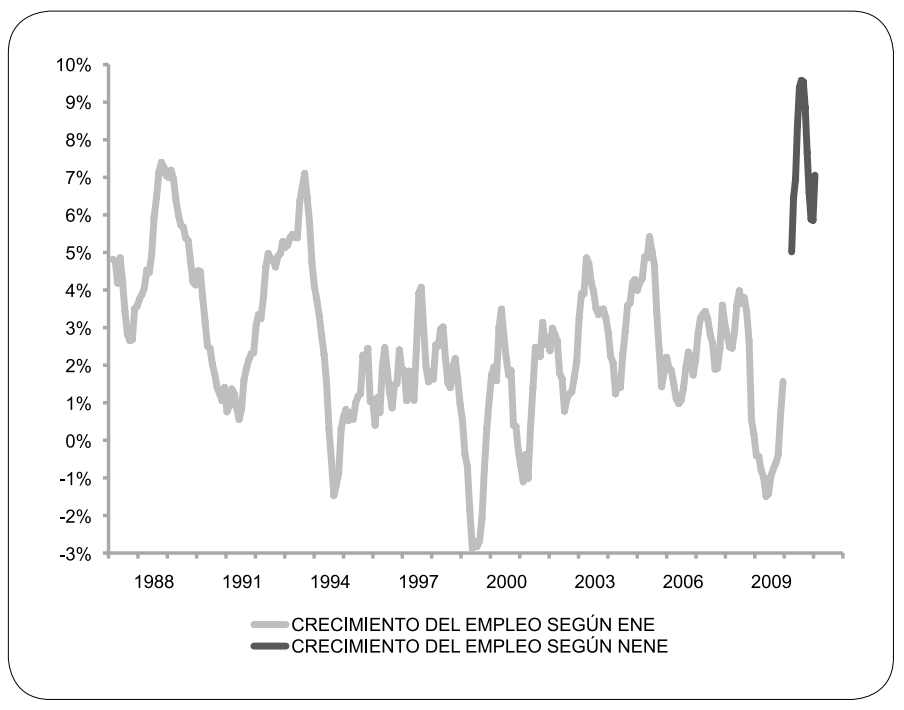

Fuente: INE

8 El error no parece atribuible a la actual Administración, porque se trataría de una decisión de la anterior Dirección del INE, enfrentada a una negativa de fondos por parte de la DIPRES del Ministerio de Hacienda (sic). Lo que si resulta reprochable a la actual Administración, es que, habiéndose recolectado los últimos datos de la anterior encuesta durante marzo de 2010, no se haya intentado continuar por un tiempo con esa encuesta para permitir un empalme técnicamente aceptable y que se den cifras de crecimiento del empleo como si no tuvieran problema alguno. 
Durante los primeros meses del año 2011, el Gobierno -en particular el Ministro de Hacienda Felipe Larraín- había omitido referirse a las cifras que se observan como promedio anual (cerca de 488.000 empleos) y había preferido mencionar 400.000 empleos como una cifra de referencia.

Ello es curioso porque no es el promedio anual que se deduce de las cifras del INE y los únicos crecimientos de esa magnitud se presentan, puntualmente, a fines de 2010 y comienzos de 2011, tal como muestra el siguiente cuadro.

Cuadro 3

Aumento en el empleo total

\begin{tabular}{|l|c|c|}
\cline { 3 - 3 } \multicolumn{2}{c|}{} & PERSONAS \\
\hline Ene - Mar & 2010 & 324.811 \\
\hline Feb - Abr & 2010 & 420.184 \\
\hline Mar - May & 2010 & 451.106 \\
\hline Abr - Jun & 2010 & 544.748 \\
\hline May -Jul & 2010 & 611.821 \\
\hline Jun - Ago & 2010 & 626.554 \\
\hline Jul - Sep & 2010 & 632.147 \\
\hline Ago - Oct & 2010 & 591.054 \\
\hline Sep - Nov & 2010 & 519.621 \\
\hline Oct - Dic & 2010 & 451.914 \\
\hline Nov - Ene & $2010-2011$ & 407.032 \\
\hline Dic - Feb & $2010-2011$ & 405.089 \\
\hline
\end{tabular}

Fuente: ENE y NENE del INE

Sin embargo, en el Mensaje Presidencial del 21 de mayo de 2011, S.E. el Presidente de la República insistió en utilizar las cifras del INE sin comentario alguno9 . En efecto el Presidente insiste en las cifras:

9 Ya habíamos hechos públicos nuestros reparos en diversas publicaciones del Instituto de Asunto Públicos de la Universidad de Chile (INAP), desde el último trimestre de 2010 y en un Seminario organizado por el INAP el 13 de mayo, en el cual comentó nuestro trabajo el Académico Harald Beyer, Subdirector del Centro de Estudios Públicos (CEP) y representante del Presidente en el Consejo Universitario de la Universidad de Chile, quien reconoció los problemas que presentan las cifras del INE. Además, los reparos se hicieron públicos en una entrevista hecha por el departamento de prensa de TVN una semana antes del Mensaje Presidencial en cuestión. 
Por eso sentimos una enorme alegría, de haber podido todos juntos, crear 487 mil empleos, la cifra más alta de nuestra historia.Y lo más importante: la mitad de estos trabajos fueron para mujeres (Piñera, 2011:6).

\section{Propuesta de recálculo de la nueva serie de empleo}

Los análisis macroeconómicos de la evolución de la economía chilena y los análisis histórico-comparativos, requieren, sin duda, un empalme menos "anómalo" que el que se observa con las series ENE y NENE en su estado actual. Para construir un indicador razonable no disponemos de suficientes cifras, a partir de comienzos de 2010 (que es cuando el INE considera “oficiales" sus registros y se hace responsable por ellos).

Ello impide separar adecuadamente los componentes de tendencia y estacionalidad de la nueva serie. Los intentos, utilizando diversos "filtros" no resultaron satisfactorios y tampoco se logró establecer una relación estable entre "niveles" de las tendencias calculadas.

De manera que sólo nos queda como alternativa, utilizar la antigua serie (ENE) cuyos datos son todos "oficiales" y ensayar una "reconstrucción" de las cifras 2009 de la nueva encuesta, utilizando para ello, los tres últimos trimestres móviles de la ENE y los trimestres móviles correspondientes en la serie NENE. Dado que se reconoce que la nueva metodología se introdujo "gradualmente”, podemos tener más confianza que los datos de la NENE, "más tardíos", hayan sido producidos con la nueva metodología "completamente" en operación:

El uso de esta metodología sencilla y fácil de replicar se fundamenta en la inexistencia de una forma técnicamente válida de calcular el crecimiento del empleo en 2010 (con respecto a 2009), porque el propio INE reconoce que no generó cifras plenamente comparables para 2009, es decir, cifras con la nueva metodología "plenamente" (y no gradual o parcialmente) operativa. 


\section{Gráfico 5}

Recálculo del periodo inicial NENE y comparación con la NENE original y con la ENE miles de personas empleadas

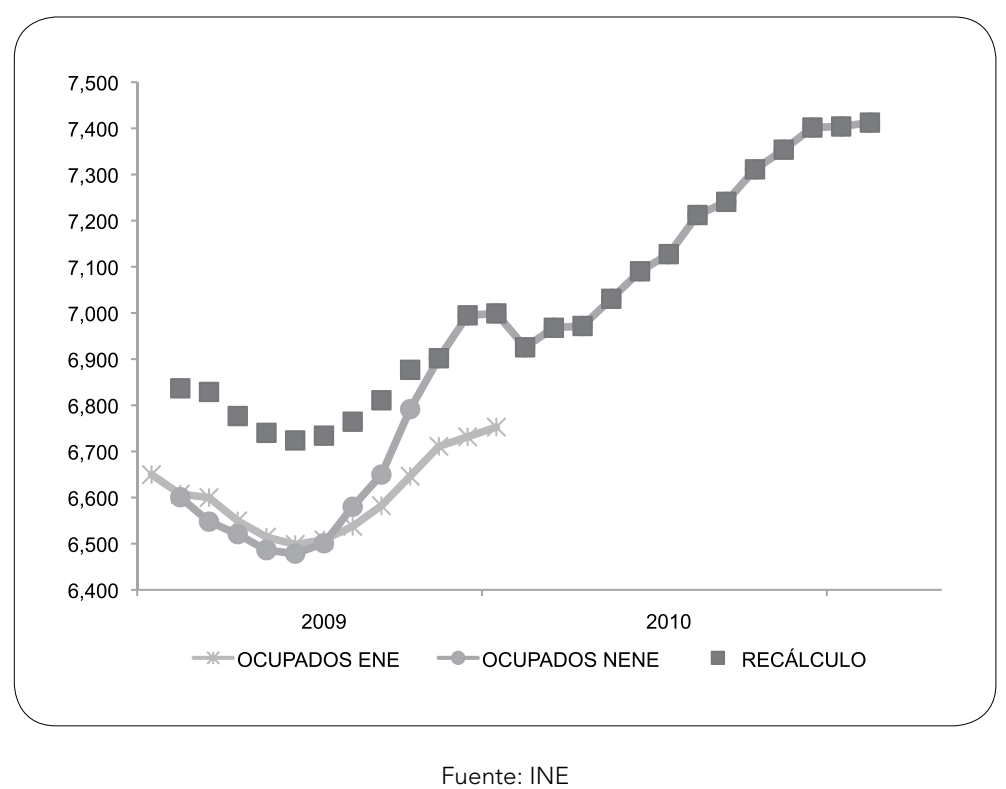

El objetivo de este recálculo no es otro que el de asumir el "salto metodológico" desde el momento inicial de la nueva encuesta (ver gráfico 5) y lograr una mayor continuidad en el comportamiento de la serie (tasas de variación anual sin saltos inexplicables, como se puede ver en el gráfico 6) y una estacionalidad similar a la de la anterior encuesta (ENE). Adicionalmente, el resultado conduce a unas tasas de desocupación que son muy similares a las que ha calculado el INE con la NENE (ver gráfico 9). Los resultados pueden verse en los gráficos 5 y 6 y en el cuadro $4^{10}$.

10 La metodología permite contar con una serie "empalmada" ENE-NENE retropolando la serie NENE con las tasas de variación de la serie ENE. La serie puede verse en el anexo. 


\section{Gráfico 6}

Recálculo de la NENE y comparación

con la serie original y con la serie antigua (ENE)

Porcentaje de crecimiento del empleo en 12 meses

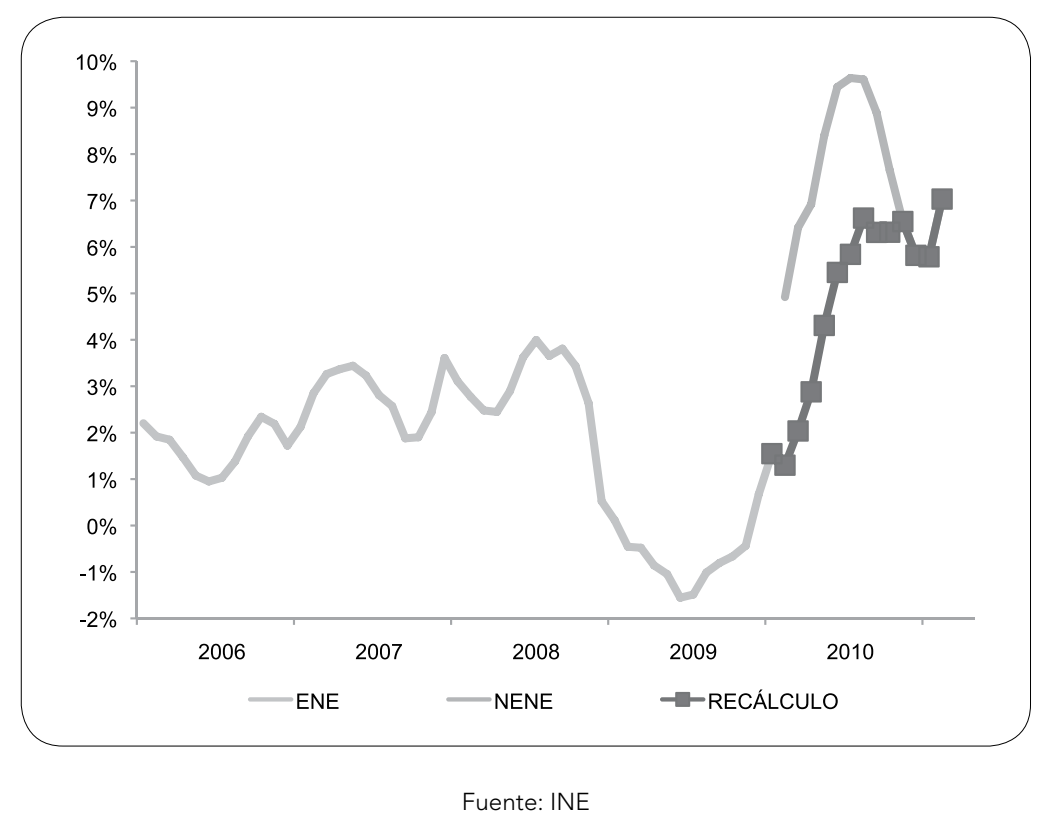

Puede observarse que el recálculo genera continuidad en el ritmo de crecimiento del empleo (gráfico 6) pero tiene la ventaja adicional de "registrar" algún efecto del terremoto en el Trimestre Móvil enero-marzo de 2010, lo cual se refleja en un ritmo de crecimiento (respecto a igual trimestre móvil del año anterior) un poco menor que lo ocurrido en el Trimestre Móvil dic. 2009 - feb. 2010 (en el cual el terremoto aún no había ocurrido) ${ }^{11}$.

11 En los ejercicios de recálculo también se consideró una "reconstrucción" de la serie NENE considerando la diferencia entre niveles en los seis últimos registros de la ENE y los registros de la NENE correspondientes. Esta alternativa, que probablemente está “contaminada”, todavía, por la introducción "gradual" de la nueva metodología en la NENE, que declara el INE, resulta en un incremento del ritmo de aumento del empleo justo en el momento en que ingresa el mes de marzo 2010 a las cifras, es decir, justo en el momento en que debería iniciarse el efecto del terremoto sobre las cifras. Este resultado poco razonable inclinó la balanza a favor de la alternativa reportada en este estudio, que utiliza los tres últimos registros de la ENE y los registros NENE correspondientes. 


\section{Cuadro 4}

\begin{tabular}{|c|c|c|c|c|c|c|c|}
\hline & \multicolumn{3}{|c|}{$\begin{array}{c}\text { Empleo en Miles de } \\
\text { Personas }\end{array}$} & \multicolumn{3}{|c|}{$\begin{array}{c}\text { Variación } \\
\text { Porcentual Anual } \\
\end{array}$} \\
\hline & & $\begin{array}{l}\text { Serie } \\
\text { ENE }\end{array}$ & $\begin{array}{l}\text { Serie } \\
\text { NENE }\end{array}$ & Recálculo & $\begin{array}{l}\text { Serie } \\
\text { ENE }\end{array}$ & $\begin{array}{l}\text { Serie } \\
\text { NENE }\end{array}$ & Recálculo \\
\hline Ene - Mar & 2009 & 6607,50 & 6600,74 & 6836,70 & $-0,5 \%$ & & \\
\hline Feb - Abr & 2009 & 6599,91 & 6547,75 & 6828,85 & $-0,5 \%$ & & \\
\hline Mar - May & 2009 & 6549,47 & 6520,69 & 6776,66 & $-0,9 \%$ & & \\
\hline Abr - Jun & 2009 & 6514,27 & 6485,94 & 6740,24 & $-1,0 \%$ & & \\
\hline May -Jul & 2009 & 6498,40 & 6478,51 & 6723,82 & $-1,6 \%$ & & \\
\hline Jun - Ago & 2009 & 6508,39 & 6500,90 & 6734,15 & $-1,5 \%$ & & \\
\hline Jul - Sep & 2009 & 6537,58 & 6580,11 & 6764,35 & $-1,0 \%$ & & \\
\hline Ago - Oct & 2009 & 6582,47 & 6649,74 & 6810,80 & $-0,8 \%$ & & \\
\hline Sep - Nov & 2009 & 6646,28 & 6791,60 & 6876,83 & $-0,7 \%$ & & \\
\hline Oct - Dic & 2009 & 6710,99 & 6901,92 & 6901,92 & $-0,4 \%$ & & \\
\hline Nov - Ene & 2009 & 6731,54 & 6994,83 & 6994,83 & $0,7 \%$ & & \\
\hline Dic - Feb & 2010 & 6752,68 & 6998,98 & 6998,98 & $1,5 \%$ & & \\
\hline Ene - Mar & 2010 & & 6925,55 & 6925,55 & & $4,9 \%$ & $1,3 \%$ \\
\hline Feb - Abr & 2010 & & 6967,93 & 6967,93 & & $6,4 \%$ & $2,0 \%$ \\
\hline Mar - May & 2010 & & 6971,79 & 6971,79 & & $6,9 \%$ & $2,9 \%$ \\
\hline Abr - Jun & 2010 & & 7030,69 & 7030,69 & & $8,4 \%$ & $4,3 \%$ \\
\hline May -Jul & 2010 & & 7090,34 & 7090,34 & & $9,4 \%$ & $5,5 \%$ \\
\hline Jun - Ago & 2010 & & 7127,46 & 7127,46 & & $9,6 \%$ & $5,8 \%$ \\
\hline Jul - Sep & 2010 & & 7212,26 & 7212,26 & & $9,6 \%$ & $6,6 \%$ \\
\hline Ago - Oct & 2010 & & 7240,79 & 7240,79 & & $8,9 \%$ & $6,3 \%$ \\
\hline Sep - Nov & 2010 & & 7311,22 & 7311,22 & & $7,7 \%$ & $6,3 \%$ \\
\hline Oct - Dic & 2010 & & 7353,83 & 7353,83 & & $6,5 \%$ & $6,5 \%$ \\
\hline Nov - Ene & 2010 & & 7401,87 & 7401,87 & & $5,8 \%$ & $5,8 \%$ \\
\hline Dic - Feb & 2011 & & 7404,07 & 7404,07 & & $5,8 \%$ & $5,8 \%$ \\
\hline
\end{tabular}

Fuente: INE 


\section{El desempeño del empleo}

Al comparar la creación de empleo entre 2009 y 2010, con base en las cifras de los cuatro trimestres de ambos años (enero-marzo, abril-junio, julio-septiembre y octubre-diciembre), con la nueva metodología NENE y con el recálculo mencionado, la creación de empleo durante el año 2010 resulta ser la que se muestra en el cuadro 5.

\section{Cuadro 5}

Comparación de la NENE y el recálculo

\begin{tabular}{|l|c|c|}
\cline { 2 - 3 } \multicolumn{1}{c|}{} & NENE & Recálculo \\
\hline Empleo 2009 recalculado & 6.642 .178 & 6.810 .802 \\
\hline Aumento en el empleo en 2010 & 488.405 & 319.781 \\
\hline Porcentaje de aumento & $7,4 \%$ & $4,7 \%$ \\
\hline
\end{tabular}

Fuente: INE

Un 4,7\% de crecimiento del empleo para todo el país es más consistente con el $4,5 \%$ que puede calcularse a partir de los reportes de la Universidad de Chile ${ }^{12}$ con una encuesta que presenta continuidad metodológica y consistencia, aunque utiliza otra metodología y cubre sólo el Gran Santiago, es decir, las comunas urbanas de Santiago más San Bernardo y Puente Alto.

En términos de promedios anuales los resultados de este ajuste son, también, más acordes con un comportamiento razonable de la productividad, dadas las cifras de crecimiento del PIB. Mientras con las cifras "referenciales" del INE la productividad laboral media cae de manera significativa (un $2 \%$ en un año), con el recálculo esta no cae (crece levemente, un $0,5 \%$ ), todo esto considerando el crecimiento del PIB que fue de un $5,2 \%$ en 2010 .

Por cierto el Gobierno del Presidente Piñera también planteó un incremento en la Tasa de Participación Laboral, buscando un incremento de ella entre las mujeres y en los hogares de mayores ingresos. Este aumento en la participación no se ha logrado (en promedios anuales), si se consideran las series recalculadas. En efecto, la Tasa de Participación Laboral promedio en 2009 es un 58,45\% y en 2010 es un 58,47\%.

Sin embargo, una vez superada la caída generada por el terremoto se observa un incremento importante hacia fines del año 2010, del orden de un punto porcentual (ver gráfico 7).

12 Ver: www.empleo.microdatos.cl/encuesta_ocupacion/encuesta-ocupacion-documentos.php?op=4 


\section{Gráfico 7}

Tasa de participación laboral en Chile 2000-2011

Según encuesta antigua (ENE) y nueva (NENE)

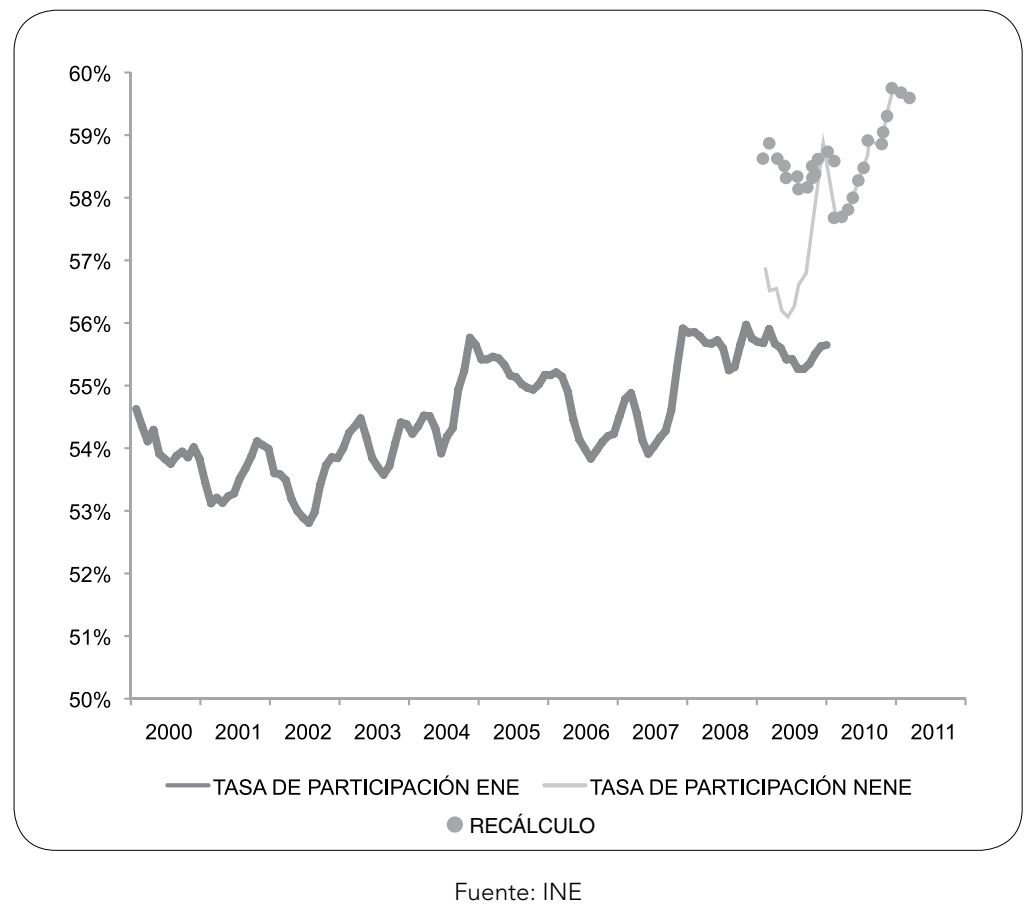

En cuanto a la facilitación del acceso a oportunidades laborales sobre la base de la posibilidad de encontrar jornadas más flexibles y promover el teletrabajo, se observa que las horas trabajadas por semana se mantuvieron bastante estables en 2010 (gráfico 8), probablemente por la presencia de fuerzas de signo opuesto (tal vez una tendencia a la recuperación de la jornada promedio, después de la crisis y una tendencia, en sentido opuesto, hacia la aparición de nuevas oportunidades de tiempo parcial).

El fomento del teletrabajo es una iniciativa que estaba en el proceso de discusión parlamentaria a fines de 2010, se le retiró la urgencia en enero 2011 para incorporarle indicaciones que se ingresaron en marzo y se encuentra, recién, en el primer trámite constitucional en la Cámara de Diputados, de manera que sus efectos sólo deberían darse en los próximos años. 


\section{Gráfico 8}

Horas trabajadas efectivas, promedio semanales: 2005-2011 La antigua encuesta nacional de empleo (ENE) y la nueva (NENE)

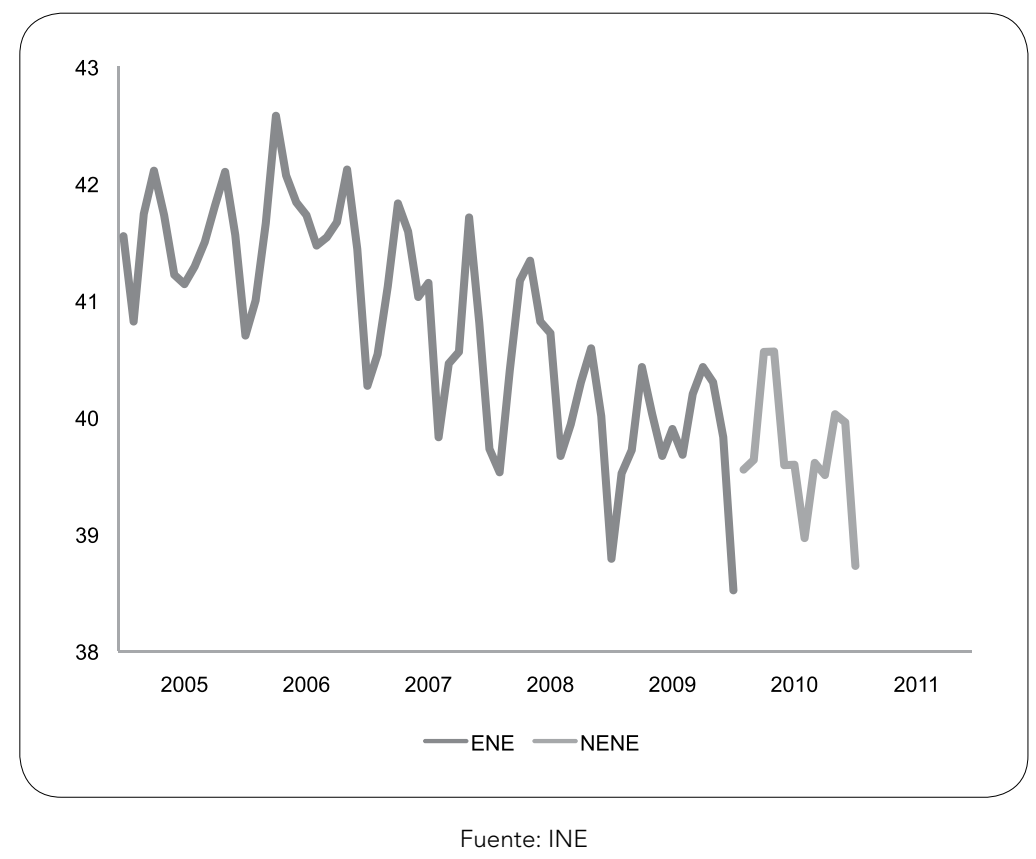

\section{Indicadores de calidad en el empleo}

El compromiso del Presidente Piñera mencionaba no sólo cantidad sino también la calidad del empleo creado. Examinaremos tres indicadores generales: los grupos de ocupación, las categorías en la ocupación y la evolución de la productividad media.

En el caso de los Grupos de Ocupación, puede observarse en el Cuadro 5 que las mayores tasas porcentuales de incremento en el empleo se registraron en el Personal Directivo del las empresas y el sector público y en los Oficiales, Operarios y Artesanos de artes mecánicas y otros oficios, mientras que en términos absolutos, un cuarto de los empleos generados corresponde a los Trabajadores no Calificados y otro cuarto a los mencionados Oficiales, Operarios y Artesanos. 
Cuadro 6

Creación de empleo según grupos de ocupación

\begin{tabular}{|l|r|r|}
\hline Total Ocupados & $7,4 \%$ & 488.405 \\
\hline $\begin{array}{l}\text { Miembros del poder ejecutivo y de los cuerpos legislativos y per- } \\
\text { sonal directivo de la administración pública y de empresas }\end{array}$ & $13,6 \%$ & 20.770 \\
\hline Profesionales, científicos e intelectuales & $2,9 \%$ & 18.996 \\
\hline Técnicos y profesionales de nivel medio & $5,7 \%$ & 40.075 \\
\hline Empleados de oficina & $2,2 \%$ & 13.885 \\
\hline Trabajadores de los servicios y vendedores de comercios y mercados & $8,7 \%$ & 89.886 \\
\hline Agricultores y trabajadores calificados agropecuarios y pesqueros & $8,8 \%$ & 22.532 \\
\hline Oficiales, operarios y artesanos de artes mecánicas y de otros oficios & $13,2 \%$ & 120.184 \\
\hline Operadores de instalaciones y máquinas y montadores & $6,4 \%$ & 37.952 \\
\hline Trabajadores no calificados & $7,3 \%$ & 121.047 \\
\hline Otros no Identificados & $6,7 \%$ & 3.078 \\
\hline
\end{tabular}

Fuente: NENE INE (sin recalcular)

En contraste con lo anterior, las tasas más bajas de creación de empleos se dan en el caso de los Profesionales, los Técnicos y los Operadores de Instalaciones y Máquinas.

\section{Cuadro 7}

Creación de empleo según categoria en la ocupación

\begin{tabular}{|l|r|r|}
\hline Total Ocupados & $7,4 \%$ & 488.405 \\
\hline Empleadores & $11,8 \%$ & 35.365 \\
\hline Cuenta propia & $15,5 \%$ & 194.490 \\
\hline Asalariado & $4,6 \%$ & 214.517 \\
\hline Personal de Servicio & $8,9 \%$ & 27.265 \\
\hline Familiar no remunerado & $19,1 \%$ & 16.769 \\
\hline
\end{tabular}

Fuente: NENE INE (sin recalcular)

Como puede verse en el cuadro 6, hubo una importante creación de empleos asalariados pero, en términos de tasas de crecimiento, se expandieron más rápidamente los Familiares no Remunerados, los Cuenta Propia y los Empleadores, ocupando el último lugar los Asalariados. Esto pone una nota de duda sobre la calidad de los empleos generados, sobre todo en el marco de un desempeño de la productividad que, como veremos, ha sido muy pobre. 
Como puede observarse en el Cuadro 8, de aceptarse las cifras "referenciales" del INE para 2009, la economía habría experimentado una considerable caída en la productividad laboral media, del orden de $-2 \%$, lo cual no ocurre con las cifras recalculadas. En efecto, al sobre-estimarse el número de nuevos empleos creados, se diluye el crecimiento del PIB entre un mayor número de personas empleadas y la productividad cae.

No es razonable atribuir una caída en la productividad a la disminución en el stock de capital generada por el terremoto del 27 de febrero de 2010. En realidad, la holgura generada por la recesión de 2009 y el ritmo de inversión existente en 2009, permitían la existencia de una brecha que hacía posible un crecimiento como el que se dio. En ese contexto el stock de capital requerido, estaba disponible (Ministerio de Hacienda, 2010b).

\section{Cuadro 8}

Productividad y empleo en Chile 2009-2010

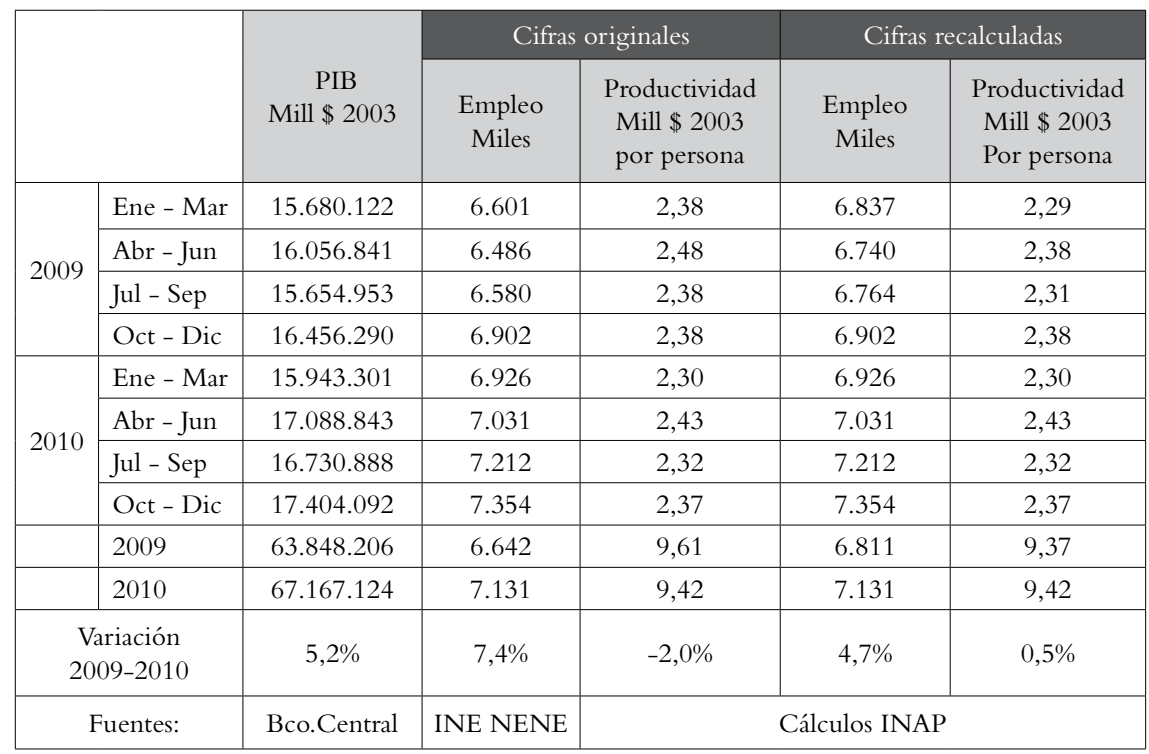




\section{El empleo y el desempleo 1990-2010}

Mirado en una perspectiva de más largo plazo, el desempeño del empleo (ocupación) y el desempleo (desocupación) puede considerarse muy positivo en 2010, aunque no constituye un año "record" como se ha pretendido.

El desempeño vigoroso registrado en las cifras de empleo, ha permitido reducir significativamente la tasa de desocupación (ver gráfico 9) hasta el punto de que se han alcanzado los niveles anteriores a la crisis internacional, aunque la nueva serie genera un tasa de desocupación alrededor de un punto porcentual mayor que la anterior (lo cual persiste con el recálculo, el cual no afecta significativamente las tasas en cuestión) de manera que podría suponerse (como una hipótesis solamente) que si mantuviéramos las mediciones anteriores la desocupación estaría alrededor de un 6\%.

No es sorprendente, entonces, que la evolución de la desocupación genere algunas aprensiones en sectores que ven en este aspecto una segunda fuente posible de presiones inflacionarias que reforzarían las provenientes del escenario internacional (altos precios del petróleo, los alimentos y las materias primas en general). De todas maneras, como a cualquier otro Gobierno, al actual se le presenta el desafio de posibilitar un ritmo de crecimiento vigoroso y sostenido, con tasas de desocupación bajas y sin que cundan las presiones inflacionarias.

\section{Gráfico 9}

Tasa de desocupación 1990-2010

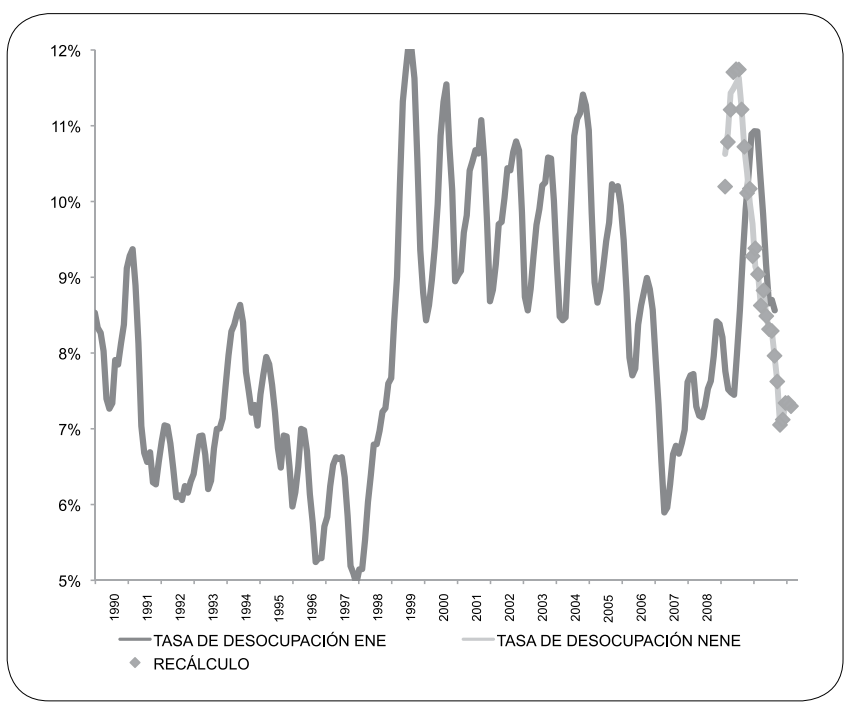

Fuente: INE 


\section{Gráfico 10}

Crecimiento del empleo y crecimiento del PIB 1990-2010

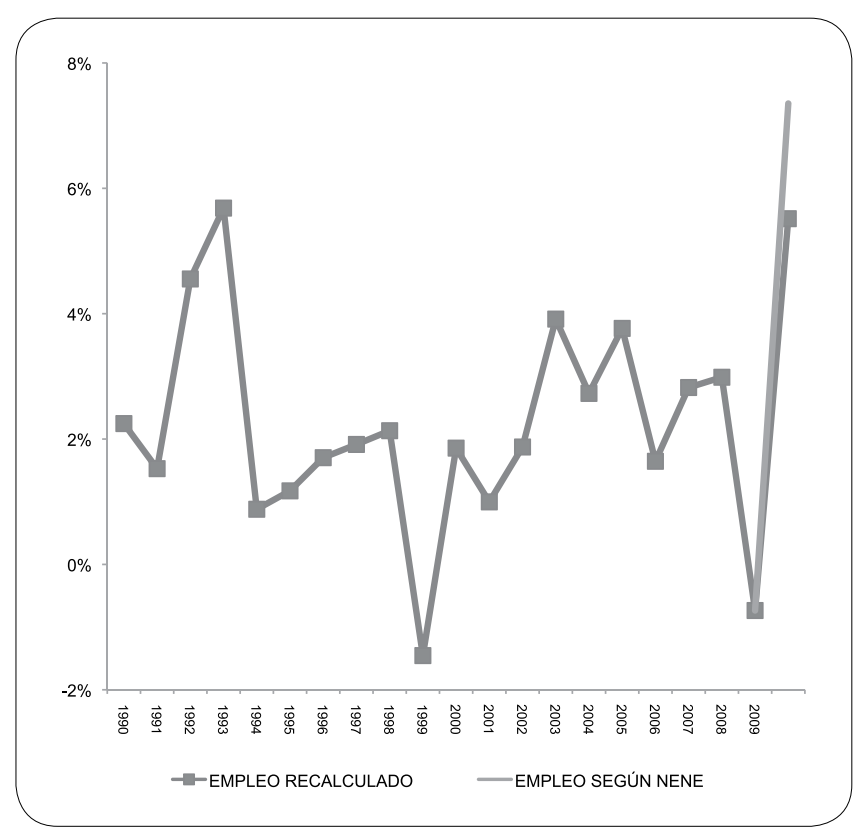

Fuente: Banco Central, INE y cálculos del Autor

En cuanto al crecimiento del empleo, este ha sido muy importante, incluso si se consideran las cifras recalculadas, pero dado que el propio Gobierno reconoce que la tendencia de la creación de empleos estará en torno a 150.000 empleos anuales si el PIB crece al 6\% anual, el recálculo implica que la meta de creación de 800.000 empleos en cuatro años sólo se cumpliría si el ritmo de crecimiento promedio se mantiene en torno a un $6 \%$ anual, logro que no puede darse por descontado (FMI; 2011).

Como puede observarse en el Gráfico 10, la creación de empleo fue muy importante en 2010 pero no constituyó un "record" (una vez recalculada), porque mirada desde una perspectiva más larga, es decir, considerando los últimos 21 años (19902010) fue un poco inferior a la de 1993 (de hecho también fue inferior a 1988 que no se incluye en el gráfico). 


\section{Referencias bibliográficas}

Albala-Bertrand, J. M. (1993). "Natural disaster situations and growth: A macroeconomic model for sudden disaster impacts". World Development, Vol. 21, Nº9, pp. 1417-1434.

FMI (2011). World Economic Outlook. Washington D.C: Fondo Monetario Internacional.

Landerretche G., Oscar (2010). "Amenazas exógenas y vulnerabilidades endógenas. Terremotos, crisis internacionales y desarrollo en Chile, 1930-2010". Estado, Gobierno, Gestión Pública, Revista Chilena de Administración Pública, Nº15-16, pp. 39-67.

Loayza, N.; Olaberria, E.; Rigolini, J. \& Christiansen, L. (2009). Natural Disasters and Growth Going beyond the Averages. Policy Research Working Paper 4980, The World Bank East Asia and Pacific Social Protection Unit \& Development Research Group.

Piñera, Sebastián (2010). "Del Chile del bicentenario al país de las oportunidades". Mensaje a la Nación de S.E. el Presidente de la República.Valparaíso: Congreso Pleno.

Piñera, Sebastián (2011). "Construyendo una sociedad de seguridades, oportunidades y valores". Mensaje a la Nación de S.E. el Presidente de la República. Valparaíso: Congreso Pleno.

Piñera, Sebastián (2009). Programa de Gobierno para el Cambio, el Futuro y la Esperanza 20102014. Santiago: Coalición por el Cambio.

Ministerio de Hacienda (2010). Agenda Chile País Desarrollado. Más Oportunidades y Mejores Empleos. Santiago: Gobierno de Chile.

Ministerio de Hacienda de Chile (2010b). Acta de resultados del Comité Consultivo del PIB tendencial. Santiago: Gobierno de Chile http://www.dipres.cl/572/articles-70119 doc pdf.pdf. 


\section{Anexo}

Serie Empalmada ENE-NENE a partir del ajuste realizado

\begin{tabular}{|c|c|c|c|c|c|c|c|c|}
\hline & 1986 & 1987 & 1988 & 1989 & 1990 & 1991 & 1992 & 1993 \\
\hline Dic - Feb & 3823,36 & 4008,71 & 4164,82 & 4466,78 & 4668,71 & 4661,07 & 4868,04 & 5122,35 \\
\hline Ene - Mar & 3833,19 & 4016,93 & 4180,89 & 4473,88 & 4644,29 & 4657,79 & 4859,44 & 5123,38 \\
\hline Feb - Abr & 3863,06 & 4025,29 & 4208,74 & 4479,94 & 4621,56 & 4629,19 & 4856,50 & 5124,59 \\
\hline Mar - May & 3843,02 & 4030,75 & 4211,40 & 4465,90 & 4577,38 & 4567,69 & 4829,74 & 5092,39 \\
\hline Abr - Jun & 3837,70 & 4001,49 & 4200,38 & 4442,64 & 4551,33 & 4527,42 & 4804,13 & 5064,78 \\
\hline May -Jul & 3834,79 & 3966,39 & 4204,37 & 4444,64 & 4533,99 & 4522,42 & 4792,73 & 5100,55 \\
\hline Jun - Ago & 3852,94 & 3961,73 & 4219,92 & 4448,75 & 4525,96 & 4550,02 & 4819,22 & 5146,28 \\
\hline Jul - Sep & 3862,28 & 3964,56 & 4250,49 & 4478,16 & 4539,49 & 4577,64 & 4840,30 & 5187,15 \\
\hline Ago - Oct & 3898,63 & 4002,92 & 4301,66 & 4507,39 & 4562,31 & 4611,11 & 4889,59 & 5208,52 \\
\hline Sep - Nov & 3940,73 & 4079,09 & 4378,37 & 4563,80 & 4610,73 & 4667,66 & 4952,53 & 5242,03 \\
\hline Oct - Dic & 3996,84 & 4140,09 & 4434,09 & 4618,25 & 4682,51 & 4741,41 & 5046,62 & 5286,52 \\
\hline Nov - Ene & 4014,99 & 4166,57 & 4460,44 & 4662,57 & 4696,49 & 4790,34 & 5091,08 & 5299,96 \\
\hline & 1994 & 1995 & 1996 & 1997 & 1998 & 1999 & 2000 & 2001 \\
\hline Dic - Feb & 5314,62 & 5356,75 & 5376,35 & 5475,71 & 5563,89 & 5590,79 & 5687,16 & 5645,96 \\
\hline Ene - Mar & 5293,43 & 5319,40 & 5378,67 & 5433,97 & 5571,96 & 5548,91 & 5656,63 & 5590,93 \\
\hline Feb - Abr & 5267,19 & 5304,65 & 5342,28 & 5439,90 & 5575,96 & 5533,91 & 5620,60 & 5597,05 \\
\hline Mar - May & 5208,11 & 5235,62 & 5337,56 & 5407,09 & 5567,39 & 5460,64 & 5624,21 & 5564,15 \\
\hline Abr - Jun & 5142,30 & 5192,10 & 5319,85 & 5375,14 & 5537,45 & 5373,41 & 5561,58 & 5578,42 \\
\hline May -Jul & 5114,34 & 5173,40 & 5269,65 & 5397,94 & 5513,89 & 5360,55 & 5510,43 & 5586,00 \\
\hline Jun - Ago & 5116,51 & 5178,55 & 5243,00 & 5449,08 & 5530,54 & 5370,05 & 5486,91 & 5622,51 \\
\hline Jul - Sep & 5107,48 & 5222,85 & 5266,33 & 5481,89 & 5557,55 & 5404,03 & 5496,98 & 5624,31 \\
\hline Ago - Oct & 5142,95 & 5239,56 & 5316,34 & 5474,28 & 5581,09 & 5459,54 & 5560,21 & 5683,74 \\
\hline Sep - Nov & 5192,13 & 5318,90 & 5398,15 & 5501,91 & 5621,09 & 5575,33 & 5595,52 & 5771,37 \\
\hline Oct - Dic & 5300,46 & 5353,90 & 5482,48 & 5566,82 & 5657,10 & 5672,99 & 5692,29 & 5837,34 \\
\hline Nov - Ene & 5331,24 & 5387,26 & 5486,58 & 5585,62 & 5638,20 & 5698,29 & 5679,01 & 5833,98 \\
\hline & 2002 & 2003 & 2004 & 2005 & 2006 & 2007 & 2008 & 2009 \\
\hline Dic - Feb & 5780,10 & 5968,94 & 6140,33 & 6386,60 & 6527,24 & 6665,84 & 6872,85 & 6880,39 \\
\hline Ene - Mar & 5757,86 & 5983,74 & 6116,82 & 6375,23 & 6497,56 & 6682,91 & 6868,09 & 6836,70 \\
\hline Feb - Abr & 5755,15 & 5980,16 & 6103,06 & 6366,61 & 6484,22 & 6695,75 & 6861,70 & 6828,85 \\
\hline Mar - May & 5711,39 & 5990,39 & 6062,96 & 6360,77 & 6454,73 & 6672,10 & 6835,31 & 6776,66 \\
\hline Abr - Jun & 5677,22 & 5946,13 & 6036,81 & 6331,29 & 6399,43 & 6619,61 & 6811,48 & 6740,24 \\
\hline May -Jul & 5676,72 & 5916,04 & 5997,73 & 6324,88 & 6384,91 & 6591,22 & 6830,00 & 6723,82 \\
\hline Jun - Ago & 5664,50 & 5889,34 & 6023,17 & 6328,49 & 6393,61 & 6573,09 & 6835,71 & 6734,15 \\
\hline Jul - Sep & 5685,48 & 5885,03 & 6055,89 & 6339,74 & 6427,29 & 6592,65 & 6833,42 & 6764,35 \\
\hline Ago - Oct & 5752,66 & 5945,46 & 6159,87 & 6369,49 & 6492,21 & 6614,15 & 6866,12 & 6810,80 \\
\hline Sep - Nov & 5844,33 & 6047,84 & 6269,05 & 6417,61 & 6567,89 & 6692,72 & 6922,86 & 6876,83 \\
\hline Oct - Dic & 5934,37 & 6142,33 & 6401,28 & 6491,24 & 6633,36 & 6795,04 & 6974,22 & 6901,92 \\
\hline Nov - Ene & 5955,05 & 6150,07 & 6414,74 & 6530,28 & 6642,65 & 6882,23 & 6918,19 & 6994,83 \\
\hline
\end{tabular}

